JOURNAL OF EXPERIMENTAL SOCIAL PSYCHOLOGY 1, 71-88 (1965)

\title{
The Requirements and Design of a Standard Group Task ${ }^{1}$
}

\author{
ROBert B. ZaJonc \\ The University of Michigan
}

Experimental work on small groups, which is steadily growing in theoretical sophistication, has been characterized by an abundance of methods and techniques unparalleled in other fields. There are nearly as many experimental set-ups and group tasks as there are small group experiments. Over the years, subjects volunteering for small group experiments have been asked to count beans (Klugman, 1945), to pull cones out of bottles (Mintz, 1951), to throw darts (Rosenthal and Cofer, 1948), to solve riddles (Shaw, 1932), to pursue a rotating target by jointly holding a steel stylus (Wegner and Zeaman, 1956), to discuss human relations problems (Timmons, 1942), to judge the merits of oriental rugs (Gordon, 1923), and even to predict the exact dates of the Japanese and German armistice before any of them were actually concluded (Klugman, 1947).

Of course, a heterogeneity of tasks in a given field of study is in principle very desirable, as it extends the generality of the findings. But the formulation of general statements from results obtained under different experimental conditions requires that these conditions be amenable to some systematic assessment and classification. An attempt to classify group tasks has, in fact, been made by Roby and Lanzetta (1958). Howcver, up to now, thcir classification appears not to have had a pronounced theoretical influence, probably because the vast majority of group tasks used in small group research simply defy a parametric analysis in terms proposed by Roby and Lanzetta, and possibly in any other terms as well.

Although social psychologists seem to prefer to construct their own group tasks for their own experiments, even though a task may already exist which is ideally suited for their particular problem, the need for a standard group task is felt by many researchers (Kelley and Thibaut, 1954; Roby and Lanzetta, 1958; Glanzer and Glaser, 1959). Some tasks have been designed which are well suited for standard uses. For instance,

\footnotetext{
${ }^{1}$ This work was supported by the Office of Naval Research, Contract NONR1224(34) NR 170-309.
} 
Roby and Lanzetta (1957) constructed an ingenious laboratory set-up which permits systematic observation of individual and group problem solving. Hall (1957) designed a task for the systematic study of twoperson cooperation. Glaser and Klaus (1961) designed a task to study the eflects of training on team proficiency. While all these tasks can be readily employed for a vast variety of experimental purposes, they have been used for a limited set of problems and only by their respective creators. In a program of research on group performance now conducted at the Research Center for Group Dynamics, a task has been designed which, hopefully, also has considerable possibilities for standard uses. Lest the reader mistake the present intention, we do not propose it as the standard task. The purpose of this paper is merely to underscore the need for standard procedures in group psychology, to examine the requirements which a standard group task should meet, and to describe our own solution in this respect.

\section{GROUP TASK REQUIREMENTS}

A useful method should always meet a set of theoretical and operational requirements. The theoretical requirements placed upon the present group task are necessarily quite broad, mainly because a coherent theory of group processes which can generate such requirements does not seem yet to exist. However, some elementary requirements might be borrowed from related fields. Inferences about group processes, like those about any other psychological processes, are ultimately based upon the observations of behavioral outputs. While individual psychologists are not always clear about what constitutes a response of an individual subject, group psychologists are even less clear about what constitutes a group response. Response rate, latency, probability, and strength of response have been well accepted and rather uniformly employed in individual psychology as standard output measures. All are intimately associated with the standard experimental situations and tasks used in individual psychology-the Skinner box, the straight alley, the maze, the pairedassociates list, and the derived set-ups. A comparable development in group psychology lags far behind. There simply is no consensus about the terms and the units which denote and measure group responses. The general primary requirement for all group tasks is then their capacity to display the behavioral outputs of the group with clarity, stability, and precision, where it can be safely assumed that these outputs are sensitive to variation as a function of variables known to influence performance.

In group psychology the clearest and strongest emphasis on the be- 
havioral outputs of the group is to be found in the study of group performance. In the study of group performance there must be some sort of product or output which is taken as a measure of performance. It is here that the problems of measuring group responses are highly criticul. It might therefore be fruitful to examine the requirements for a group task on the basis of requirements for the systematic analysis of group performance. With this view in mind a set of criteria and specifications was developed, which a group task for continuing and systematic experimentation on group performance should exhibit. We shall spell out these requirements.

In the last analysis group performance depends directly on two classes of factors: (a) the performance of individual members, and (b) the pattern of task assignments. All other variables affect group performance only through acting upon one or both of these primary classes of factors. Thus, one can change group performance if one can change the performance of individuals working as a group, or one can change it by distributing the individual task assignments or loads in a new way, or by both methods. The requirements for a group task enumerated below have been specified with these considerations in mind.

\section{Antecedents of Individual Performance}

Quite frequently group tasks involve behaviors about which there is minimal systematic knowledge. While there is a modest amount of reliable psychological information about certain kinds of individual behavior, we are still quite ignorant about factors affecting the performance on an anagrams task (e.g., Faust, 1959), the performance in basketball (e.g., Fiedler, 1954), or coal mining (e.g., Trist and Bamforth, 1951). The interpretations of the results of experiments with such tasks remain tenuous, and we often find the results to contradict one another (Kelly and Thibaut, 1954). Clearly, precise statements about group performance cannot be made in the absence of systematic knowledge of the performance of individual members which contribute to it or which make it up. We feel, therefore, that a standard group task should involve individual behavior reasonably well understood, behavior extensively examined in individual psychology, behavior whose antecedents are reasonably well known. For instance, it should be quite clear before experimentation on the group level how such factors as stimuli, motivation, stress, load, practice, and the like affect individual performance; what is the range of individual differences to be expected; how such behaviors are acquired; how they are affected by reinforcement; and how stable is the asymptotic performance level. 


\section{Group Task and Individual Range of Talent}

Many tasks used in the past have required considerable intellectual development, at least that characteristic of college sophomores. A task which can be used with any population would, of course, be morc desirable. We feel, therefore, that a standard group task should involve individual responses which are common to or may be quickly acquired by any population.

The Assessment of Individual Ability and Performance Outside and Independently of the Group Situation

Quite often group experiments involve individual behavior which cannot be assessed outside and independently of the group situation. For instance, the relative skills with which different individuals are able to discuss human relations problems cannot be assessed outside and independently of the group situation. Group problem-solving and group decision-making are also of this variety. Just what constitutes the units of performance of individual members in tasks of this type is difficult to determine, and thcrefore difficult to measure. A standard group task should, therefore, allow the precise preferably quantitative and independent assessment of individual capability and of individual performance in measurement units commensurate with units used to assess group performance. Such a task would facilitate the observation of various effects upon individual performance in groups and the determination of individual contributions to the group product.

\section{The Contribution of Individual Members to the Group Product}

It has been a serious difficulty in the systematic investigation of group performance that the contributions of individual members could not always be precisely specified. For instance, if one considers, in a basketball game, the performance of the team in terms of the points it was able to score against its opponents, these points are in fact the sum of points scored by individual players. On the other hand, however, a basket sunk by an individual does not represent the consequence of his and only his ability and effort, but to a large extent is also a team product. Il would thus be very difficult in experimental group situations of a similar character to determine independently the contribution to the team product made by each individual. Many tasks in the literature on group performance are characterized by such a shortcoming. A group task should enable the experimenter to evaluate individual contributions independently of the evaluation and measurement of the group performance, but in terms commensurate with those used to assess group 
performance. Moreover, the experimenter should have the ability to arrange the task so that he could experimentally manipulate these individual contributions.

\section{Performance Interdependence among Individuals}

Group tasks used in social psychology range from those in which individuals are quite independent of one another to those in which behavior is highly interdependent. Clearly, if a task is to be useful in the systematic research on group performance it should allow the free manipulation in terms of such interdependence, without at the same time changing its basic character or that of the individual responses involved.

\section{Individual Task Parameters}

Among the task parameters which the experimenter could vary, individual task difficulty or probability of individual success, task complexity, and knowledge of own and others performance are of prime consideration.

\section{The Measurement of Group Performance}

It is perhaps obvious to require that group tasks be amenable to measurement in terms of objective, quantitative, and meaningful units. Such criteria as the number of puzzles solved, or the amount of fuel used in landing an aireraft, are often too indirect and therefore subject to variation as a result of factors of no interest to the experimenterfactors which should ideally be held constant. In general, a direct, quantitative, and standard measurement of the group product should greatly facilitale research when a series of experiments on a series of related problems is contemplated. Moreover, the units of measurement of the group product should be commensurate with those employed in the assessment of individual performance, so that one could readily evaluate the contributions of individual members to the group product.

\section{Task Assignment Patterns}

Besides individual abilities and individual performance, the outcome of the group efforts depends on the way in which the group task has been subdivided among its members. The problem of optimal task assignments is of considerable importance in the study of group performance (Zajonc and Smoke, 1960), and the group task should be flexible in allowing many varieties of division of labor. Both, assignments that assign heterogeneous as well as homogeneous task units to members, should be possible. The latter would probably be necessary in the study 
of problems of coordination of differentiated individual effort. These manipulations should be possible without disturbing the individual behavior involved.

\section{Group Task Parameters}

As in the case of individual task parameters, the group task should also be capable of manipulation in terms of diffculty, probability of success, complexity, and achievement or performance feedback.

\section{General Task Requirements}

In addition to the above considerations a standard group task should also exhibit some characteristics which are generally desirable, that is, characteristics applicable for all standard tasks. For instance, economy in collection of data is often of serious importance in programmatic research. A task which one hopes to use repeatedly should allow one to collect data within a minimum amount of time, but simultaneously, should guarantee a fair stability of observations. Also, the task should be useful for the study of a large variety of problems in the area of group performance without requiring drastic task changes. If changes are required they should never affect the units of measurement used to assess individual and group performance. Finally, it should be possible within the realm of task facilities to obtain repeated measurements of the same behavior from the same individual under the same or different experimental conditions. This is often not possible, as for instance, when the group task consists of solving puzzles. The same puzzle cannot be administered to the same individual in successive experimental situations. The second time the subject is given the puzzle he already knows the answer to it. Consequently, when we are interested in observing what happens to. individual performance in group situations, and when we wish to compare the performance of an individual as he works alone and as he works in groups, we require a task on which performance could be observed independently and repeatedly. It would also be useful to have a task which is not confined to a particular laboratory installation but which could also be administered outside the given installation. A portable experimental set-up has some obvious advantages over a bolted-down set-up.

\section{GROUP TASK DESIGN}

A standard experimental procedure has always proved superior to ad hoc methods. However, in reviewing the literature on group performance one realizes that such tasks are difficult to find. The closest approximation to the above requirements is the task designed by Roby 
and Lanzetta (1957). While the Roby-Lanzetta task is very flexible and has many other advantages, it has the drawback that the individual behaviors required of subjects have not been extensively studied and are not too well understood. The subjects in their task are required to set dials at certain points. The group task to be described below involves individual reaction times. We shall first describe the apparatus itself, its operational features, and finally, discuss the experimental opportunities which the task offers.

The apparatus consists of seven individual panels and a control console. The panels are operated by the subjects, and the present set-up permits experimentation with groups of up to seven persons, although the circuit $^{2}$ can be expanded to accommodate groups of any size. An illustration of an individual panel is shown in Fig. 1. The panels are the size of a portable typewriter, and they can be placed either in separate cubicles, or on a large table on which partitions have been built to prevent subjects from seeing each other, or, of course, with the partitions removed, when one wishes the subjects to see each other and each other's panels.

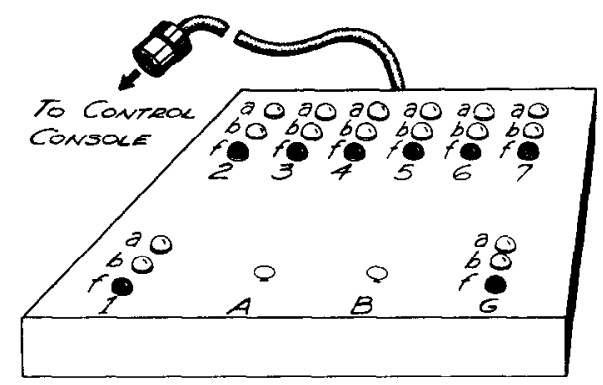

Frg. 1. An illustration of individual working panels.

Normally, the subjects are required to turn off, as quickly as possible, a light which appears on their panels. Each panel contains two reaction keys, marked $A$ and $B$, and eight stimulus displays. One of the stimulus displays, located always on the lower left of the panel, is the subject's own display. A display marked $G$ which is located in the lower right corner is a "group" display, to which subjects respond as a group. The remaining seven displays provide feedback about the behavior of other group members.

Each stimulus display consists of two stimulus lights, $a$ and $b$, and a red "failure" light, marked $f$. The action of each key can be coordinated to either of the two stimulus lights. Thus, one can set the apparatus so

${ }^{2}$ A circuit diagram of the Group Reaction Time Apparatus and a parts list may be obtained from the author on request. 
that key $A$ turns off light $a$, or that it turns off light $b$. The same applies to key $B$. This allows working with simple reaction times, involving : single response to a single stimulus, as well as more complex behavior involving any combination of two stimuli and two responses.

Stimulus lights $a$ and $b$ are turned on by the experimenter following a warning interval which can be automatically regulated. They are turned off by the subjects (in a particular pattern or according to a preset criterion which will be described shortly) by pressing the appropriate keys. The so-called "failure" lights are set by the experimenter for any time interval following stimulus onset. Normally, if the subjects respond before the failure light should go on, their stimulus lights go off, and failure lights do not go on. If they fail to make the appropriate response before that time, the failure light does go on, and they are unable to turn the stimulus light off.

The feedback about the performance of other group members, about the group performance as a whole, or about the subject's own performance, can be either fully given, fully or partially withheld, or falsified.

To create a group task the response keys of the group members are connected in various ways to form a great variety of group task patterns. Each key is connected to a reaction clock which registers reaction times of the individual subjects. There is also a reaction clock to time the group response. Normally, before the experiment, subjects are tested individually over a series of trials to determine their particular average reaction times as they work alone. A stable baseline may be obtained after less than fifty responses, or in about 10 minutes. Data collected subsequently in the group situation can thus be evaluated against the baseline previously obtained.

\section{Specific Experimental and Operational Options}

1. Data may be collected in the form of milliseconds as well as in terms of success and failure. Consider a frequency distribution of reaction times of a given subject plotted against time elapsed since stimulus onset, such as shown in Fig. 2. By setting the failure timer appropriately, the experimenter determines the subject's probability of success. Setting the failure time at the median of the distribution results in a probability of success of .5 . Of course, any other values can be arbitrarily chosen to manipulate task difficulty.

2. Given a number of subjects (in the present apparatus up to seven), the probability of success can be equalized across the subjects. Even though the average reaction times of all subjects differ, one can set the failure times, for instance, at each subject's own median, thus cancelling all individual differences in probability of success. Moreover, by means of 
differential settings of the failure timers, any distribution of individual probabilities of sucecss (or vicwed from another vantage point, individual apparent abilities) can be achieved. Thus, one can have situations in which some members succeed very frequently, while others succeed seldom or even never.

3. Several options are available by manipulating the failure light. The apparatus enables the experimenter on any given trial to set the failure light so that it will light and inhibit the offset of the stimulus light regardless of the subject's response. Thus, we may set the failure time at the subject's median reaction time, and by the adjustment of proper controls on the control console, make the subject believe that he failed even though he may have in fact succeeded. If the subject's median reaction time is, for instance, 180 mseconds, and we set the failure time for 180 mseconds, normally, the subject will succeed $50 \%$ of the time. However, by bypassing the subject's responses and activating the failure light by the timer, the failure light will go on at 180 mscconds following stimulus onset and inhibiting the offset of his stimulus light, regardless of whether the subject reacts before or after 180 mseconds have elapsed. Since the subject's performance will vary between approximately 160 and 200 mseconds, he is in fact unable to determine whether he has responded in time, because the time intervals are much too short for him to discriminate.

It is also possible to inhibit the failure signal such that it will not go on, regardless of the subject's performance, thus making him believe that he has succeeded when he actually failed. These manipulations were recently carried out in a series of studies designed to examine the effects of changes in apparent performance of group members on the role

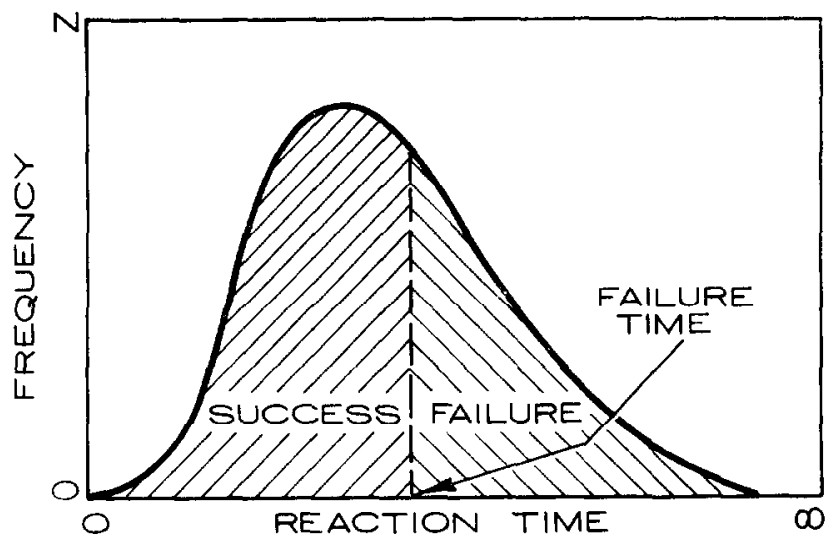

Fig. 2. The definition of reaction time in terms of success or failure. 
structure of the group (Burnstein, Zajonc, and Taylor, 1963b). The false failure feedback was successfully maintained over a series of 60 trials. Individuals who initially appeared to other group members and to themselves to perform at a high level were gradually shifted to a low level of apparent performance, simply by manipulating the onset of the failure signals. In other groups, members initially slow in the eyes of the group and in their own, have been presented as gradually improving. The actual performance of these members was observed during these manipulations, and it was shown that performance is strongly affected by changes in apparent performance.

4. Normally, the object of the group task is for the group to turn off, as rapidly as possible, the group stimulus light (marked $G$ on the individual panels, Fig. 1). The way the group can achieve this can be manipulated in various ways:

a. The individual reaction keys of the members may be connected so that the group stimulus light is turned off when at least one individual reacts in time, i.e., before the onset of the failure light. The group product can also be defined simply by the time when the first individual reacts, ignoring the failure signal altogether.

b. The reaction keys may also be connected so that the group stimulus light is turned off when at least two, three, four, five, or six members react in time. Under these conditions group performance depends on a specifiable quorum varying from a small minority to a substantial majority.

c. Of course, the reaction keys may also be connected so that the group succeeds if and only if all individual members react in time. If the probability of individual success is held constant at $p$, then the above variations allow the manipulation of the probability of group success ranging from $1-(1-p)^{7}$ to $p^{7}$. Holding the criterion of group success constant, let us say for instance, by requiring at least four individuals to react in time, one can also vary the probability of group success by adjusting the probability of individual success, which is accomplished by appropriate setting of the failure time. It should be noted that the above manipulations of the probability of group success (or group task difficulty) are independent of one another. In a recent study (Zajonc and Taylor, 1963) it was shown that both individual and group performance are more sensitive to variations in group task difficulty when the former method of varying the probability of group success is utilized. It is, of course, also possible by a proper adjustment of the probability of individual success, $p$, and of the criterion of group success, to hold the probability of group success constant while varying individual task assignment difficulties and group success criterion. It is 
clear that by gradually increasing the criterion of group success from the least stringent (i.e., group suceceds when at least onc momber reacts in time) to the most stringent (i.e., group succeeds when all members react in time) and by simultaneously and appropriately increasing the probability of individual success, $p$, the probability of group success will remain constant. It would be of interest to determine how such manipulation affects individual and group performance.

5. Other options in defining the group task are also available. For instance, one can have the group succeeding if and only if a particular group member reacts in time, or when a particular subset of members react in time. It is equally possible to define group performance in terms of the sum of individual performances, i.e., the sum of all individual reaction times. Various forms of the group response were recently specified and analyzed (Smoke and Zajonc, 1962), and all of them can be obtained with the Group Reaction Time Apparatus.

6. The above manipulations of the criterion of group success already involve some degrees of interdependence among the group members. Thus, when group performance is coordinated to the sum of the performances of all the members, each member contributes to the group score. If, however we define the group score as the time at which at least one individual member reacted, then the group score depends entirely on the performance of the fastest group member. If we define the group score as the time at which all individual members reacted, then the score depends entirely on the slowest group member. In addition to these and derived forms of interdependence, others are also possible. For instance, it is possible to produce a condition where the offset of the stimulus produced by one subject can serve as the onset of the stimulus for another subject, whose stimulus offset then lights the stimulus light for a third subject, and so on. Under these conditions the interdependence is sequential. Also, the group members may be required to use various patterns of key responses. For instance, the experimenter may require the group to use the same key as the first subject used, or to alternate keys, or to double alternate, and so on. The distinction between goal and task interdependence introduced by Thomas (1957) is readily operationalized by the present set-up. Task interdependence can be manipulated by setting the reactions in a required sequential arrangement as suggested above, where the offset of one stimulus initiates the sequence for another subject. The sequence may be constant for all trials, or the pattern may be varied over trials so that on trial $n$ subject $\mathrm{Z}$ follows subject $\mathrm{Y}$, and subject $\mathrm{Y}$ follows $\mathrm{X}$, while on trial $n+1$ subject $\mathrm{Y}$ follows $\mathrm{Z}$, who follows $\mathrm{X}$, etc. Moreover, the task difficulty of subject $Z$ may be set to depend on the 
performance of subject $\mathrm{Y}$ who preceded him. Thus, we may wish to increase the probability of success by setting the failure time for subject $\mathrm{Z}$ for a longer interval when subject $\mathrm{Y}$ had just reacted very rapidly. or we may want to decrease $Z$ 's probability of success by the same method. Goal interdependence is operationalized by manipulating the distribution of the group achievement among the individual members. Thus we may divide the group score equally among the members, independently of their own contribution to it, or the individual's score may be defined as a weighted sum of his own performance and that of the group as a whole. Increasing the weighted group component of the individual scores increases goal interdependence among the individuals.

7. As already noted above, feedback regarding performance may be freely manipulated. The group may operate with full feedback. Here the subjects are fully informed about each member's success and failure, their own reaction times, and the achievement of the group as a whole. Any element of this full feedback may be freely withheld over a series of trials, on a single trial, for all subjects, or for some subjects only. In a recent study (Zajonc, 1962), the effects of total feedback and socalled confounded feedback on individual and group performance were compared. In the total feedback the subjects are given full information regarding each member's contribution to the group product in addition to also having information about whether the group as a whole succeeded or failed. In the confounded feedback condition the subjects only know whether the group failed or succeeded. Both group and individual performance was found to suffer under confounded feedback. Differences were also found in the way that feedback affected group members who are relatively slow and members who are relatively fast. In the above experiment the information given to subjects was accurate. However. feedback can also be distorted so that subjects are given the impression that they are succecding when they are actually failing, or that they are failing when actually succeeding (Burnstein et al., 1963a,b).

8. Several experimental problems in the area of group performance require the manipulation of division of labor. The Group Reaction Time Apparatus allows such manipulation readily. We shall give examples of two possible ways of varying division of labor. On the simplest level one can vary division of labor by simply varying the task loads of individual members. This manipulation is accomplished by either making probability of success to vary across the individual members, such that in some conditions all individuals are subject to the same probability of individual success (i.e., have equal task loads), or such that the probability of individual success has a high variance across the group members. In this example division of labor is manipulated simply along 
the quantitative dimension, i.e., the main concern is with the distribution of task loads. Of some interest also are the effects of division of labor on performance where division of labor is conceived in terms of substantively differentiated functions. A primitive form of such division of labor was implied above when we suggested that interdependence among members may be accomplished by connecting the response keys of the individual group members into a relay system, such that the stimulus offset of one member becomes the stimulus onset for another. Under these conditions, as in the track relay, the responsibilities of the first man differ from those of the last man. More complex varieties of division of labor may be instituted by requiring a more complex behavior of the experimental subjects. Suppose we have two individuals and we employ a two-stimulus and two-response reaction measure. Moreover, the stimuli are set such that one goes on $70 \%$ of the time and another $30 \%$. We may have one group member being responsible for actually deciding which of the two stimuli will go on the next trial, and the other group member doing the physical work of reacting. Of course, with a greater number of people and with a more differentiated decision process, such division of labor may be further differentiated.

9. Once there exists a means of manipulating division of labor, the experimental set-up allows the possibility for the study of role development. The various task assignments can be specified at the outset of the experiment not for particular group members but for the particular panels. Thus, for instance, some panels may be given a failure time of 200 mseconds (easy task) while others a failure time of 150 mseconds (difficult task). We may then let the group decide which individual member will be assigned which task and which panel. By letting the group operate in this manner over a series of trials, the opportunity may be given to them to change these assignments, and the stability of the role assignments may thus be observed under various conditions. In one series of studies observations were made on the development and stability of such role structures when the group success was manipulated (Burnstein et al., 1963a).

10. The effects of such variables as cooperation-competition on individual and group performance can be readily observed. The simplest set-up involves the use of two sets of three or two panels, where each set constitutes a team. The teams compete for the fastest group reaction time (or the greatest number of success), while the group members within each team constitute a cooperative unit. Competition among individuals is achieved by simply announcing that the individual with the fastest reaction time will receive some sort of reward.

11. Many experiments in group performance involve complex be- 
haviors such as problem-solving. The Group Reaction Time Apparatus may be readily used for the study of group and individual problcmsolving. The seven reaction keys can be wired to form a sequential pattern (for instance, $3,6,5,1,2,4,7$ ) such that only the given sequence of responses will turn off the group stimulus light $G$. We may then let the group discover the pattern and observe the process by which it arrives at the solution. Of course, more complex patterns may also be constructed.

12. An entire host of variables which in the past have been studied in relationship to group performance can be readily manipulated. Such variables as stress, social facilitation, group composition in terms of personality or other individual differences, external reference norms, incentives, motivation, group size, and the like, can be as easily manipulated in the context of the group reaction time set-up as in any other experimental group task.

\section{Application of the Group Reaction Time Apparatus to Problems Other than Group Performance}

In many problem areas of social psychology individual and group performance constitute the dependent variables, but the interest is not in performance per se but in another factor of which performance is taken as an index. This is the case, for instance, when we study conformity, group pressures or standards, imitation, or vicarious reinforcement. In other problem areas individual and group performance are used as the independent variable, as for instance in studies on level of aspiration. Still in other areas individual and group performance are neither the independent nor the dependent variable but are required to obtain measures of the variables under investigation, as for instance, in the study of leadership, interaction process, or role structure. The Group Reaction Time Apparatus is also useful for these cxperimental problems. We shall give illustrations of a possible application of the Grouj Reaction Time Apparatus in each of these three classes of problems.

For the study of imitation, the simplest procedure would be to require one of two subjects to respond on the basis of the response of the other (model). We may want to use two reaction keys, and program the success feedback in such a way that the follower "succeeds" it and only if he reacts after the "model" has chosen a response, and when he responds with the same key as the "model." For vicarious reinforcement only one of two individuals who are in full view of each other is reinforced for a particular pattern of responses, and we make observations on the behavior of the critical subject. The study of conformity or of the effects of group standards is possible by providing a 
false feedback to the critical subject, which makes it apparent to him that for one reason or another the remainder of the group is consistently slowing down or speeding up in their reactions. Observations of the performance of the critical subject can then be made. This paradigm clusely approximates the sort of siluation which is of consequence in industry where the tempo of work is governed by some group standards (see, for instance, Coch and French, 1948).

The Group Reaction Time Apparatus has a particular advantage in the study of level of aspiration. In the majority of instances the level of aspiration paradigm requires on each trial that the subject commit himself to an expected performance level and that he subsequently complete the performance. The actual performance of the subject following his commitment is clearly visible to him, and subsequent commitments are apparently determined by his past performance. Thus the actual performance of the subject is a critical variable in determining his level of aspiration. In spite of the fact that actual performance is of such critical importance in level of aspiration studies, it has never been experimentally manipulated. The reason for this is that the subjects' performance could not be controlled. With the Group Reaction Time Apparatus this is possible. We allow the subject to work in blocks of ten trials. We set the failure time at some pre-arranged time interval and require the subject to commit himself as to how many successes out of the ten forthcoming trials he expects to attain. Since it is possible to manipulate the number of apparent successes by falsifying the failure feedback, the subject can actually be given controlled, albeit false, information about his performance. The time intervals here are so minute that it is very unlikely that any of the subjects would detect the deception. This experimental set-up allows the study of the effect of performance on level of aspiration. Of course, level of aspiration studies also examined this relationship. However, there is a basic difficulty with such an analysis as performed in the past. On the one hand, it is assumed that level of aspiration depends on the subject's past performance. On the other hand, level of aspiration is usually altributed motivational properties, and it has therefore some influence upon the performance of the subject. Since the two variables were in the past experimental designs confounded, a clear statement of the influence of one upon the other could not be obtained.

In discussing the operational options for the manipulation of division of labor, some possibilities of studying the development of a role structure were already suggested. A study with the Group Reaction Time Apparatus on the stability of role structures was recently carried out (Burnstein et al., 1963a). Each of four panels was assigned a number 
of points from 20 to 100 , and these points could be contributed by the subject at that panel to the total group score, providing the occupant of the given panel succeeded on the given trial. After the collection of data on their baseline reaction time performance, four subjects were assigned to these four panels, such that the correlation between their baseline reaction times and the panel payoff was - 1.00 , that is, the fastest member could contribute the greatest number of points to the group score, and the slowest, the smallest number of points. After each block of five trials the group was given the opportunity to vote on whether to maintain the present distribution of panels among the members, or whether to change it. When the group voted for a change, the subjects discussed the re-assignment, and after agreeing on the new distribution of panels, the panels were actually re-assigned. After four blocks of trials the success feedback in some groups was gradually falsified so that it appeared that the originally fastest member slowed down to a point where he was now the slowest. In other groups the feedback gave the impression that the slowest member improved so much that he surpassed the originally fastest member. In control groups, no such changes in the feedback occurred. Among others, it was observed that group members are extremely sensitive to such changes in apparent performance in that they vote for a re-assignment and actually change the assignment of panels. The probability of group success was also manipulated so that in one condition the group succeeded on all trials, and in another it succeeded on only $40 \%$ of trials. It was found that the changes in re-assignment of pay-offs occurred more rapidly under conditions of complete success than under conditions of limited success.

\section{CONCLUSIONS}

Although it is not the intention of the present paper to suggest the Group Reaction Time Apparatus as the standard experimental task for problems in group psychology, its advantages are worth underscoring. In our research experience the Apparatus has proved to be a flexible experimental set-up, allowing the study of various problems under systematically controlled conditions, and above all, providing stable and reliable results. The economy of the data collection procedures is truly remarkable, for it is easy to make 1500-2000 observations within at single hour. The task itself is simple and the behavior of individuals in the group is also quite simple. The generality of the results will, of course, not extend over the entire range of performances which groups may engage in. Clearly, results obtained with the Group Reaction Time Apparatus may not be directly applied to explain the differences between the performance of symphony orchestras, football teams, industrial 
organizations, military units, or high trapeze troupes. Such a method does not exist at the present time. But, on the other hand, the task guarantees some very important advantages: constancy of individual responses under varied task parameters, economy and precision in data collection; above all, it allows comparisons of results about group performance obtained under different conditions.

The Apparatus is not very expensive, by the present standards. Parts and labor amount to approximately $\$ 1500$. If more precise reaction time measurement is desired, electronic reaction clocks may be replaced for the electrical ones now employed, thus increasing the cost of the apparatus by $\$ 3000-\$ 4000$. The maintenance of the equipment is minimal after the initial defects in the circuit have been located, and if the original construction was performed competently.

In closing, it might also be worth noting that the Group Reaction Time Apparatus, because of its flexibility, constitutes a very effective teaching aid in experimental social psychology courses. The students can collect a relatively large amount of data on a variety of problems, in a relatively short time. Since the experimental set-up makes certain problems of experimental design, measurement, and control dramatically clear, the students gain valuable research experience while working out these problems directly rather than abstractly.

\section{REFERENCES}

Burn STEIn, E., ZaJonc, R. B., AND TAYLOR, J. J. The effect of apparent, performance. and group success on changes in status within a group structure. Tech. Rep. 22, ONR-1224(34)NR 170-309. Ann Arbor: Research Center for Group Dynamics, 1963a.

Burnstein, E., Zajonc, R. B., ANd TAYlor, J. J. The effects of status on group and individual performance. Tech. Rep. 23, ONR-1224(34)NR 170-309. Ann Arbor: Research Center for Group Dynamics, 1963b.

Coch, I., ANd French, J. R. P., Jr. Overcoming resistance to change. Hum. Relat., $1948,1,512-532$.

Faust, W. L. Group versus individual problem-solving. J. abnorm. soc. Psychol., $1959,59,68-72$.

FiEDLER, F. E. Assumed similarity measures as predictors of team effectiveness. J. abnorm. soc. Psychol., 1954, 49, 381-388.

Glanzer, M., and Glaser, R. Techniques for the study of group structure and behavior: I. Analysis of structure. Psychol. Bull., 1959, 56, 317-332.

Glaser, R., and KlaUs, D. J. Laboratory studies of team training. Amer. Psychol., 1961, 16, 586. (Abstract)

Gordon, K. A study of esthetic judgments. J. exp. Psychol., 1923, 6, 36-44.

Hall, R. Group performance under feedback that confounds responses of group members. Sociometry, 1957, 20, 297-305.

Kifluer, H. H., and Thtbaut, J. W. Experimental studies of group problem solving and process. In G. Lindzey (Ed.), Handbook of social psychology. Cambridge: Addison-Wesley, 1954. 
Klougan, S. F. Group judgments for faniliar and uniamiliar materials. J. gen. Psychol., 1945, 32, 103-110.

Klugmas, S. F. Group and individual judgments for anticipated events. J. soc. Psychol., 1947, 26, 21-28.

Mintz, A. Non-adaptive group behavior. J. abnorm. soc. Psychol., 1951, 46, 150-159.

RoBY, T. B., AND LANZETTA, J. T. A laboratory task for the study of individuals and groups. Tech. Rep. AFPTRC-TN-57-124, ASTIA AD 134 256, Air Forec Personnel and Training Research Center, 1957.

Roby, T. B., AND LANZETTA, J. T. Considerations in the analysis of group tasks Psychol. Bull, 1958, 55, 88-101.

Rosenthal, D., and Cofer, C. N. The effect on group performance of an indifferent and neglectful attitude shown by one group member. J. exp. Psychol.. $1948,38,568-577$.

Shaw, Marjorie E. A comparison of individuals and small groups in the rational solution of complex problems. Amer. J. Psychol., 1932, 44, 491-504.

Smuke, W. H., and Zajonc, R. B. On the reliability of group judgments and decisions. In Joan Criswell, H. Solomon, and P. Suppes (Eds.), Mathematical methods in small group processes. Stanford: Stanford Univer. Press, 1962. Pp. 322-333.

Timmons, W. M. Can the product superiority of discussers be attributed to averaging or majority influences? $J$. soc. Psychol., 1942, 15, 23-32.

Trist, E. L., ANd Bamforth, V. Some social and psychological consequences of the longwall method of coal-getting. Hum. Relat., 1951, 4, 3-38.

Wegner, Norma, and Zeaman, D. Team and individual performances on a motor learning task. J. gen. Psychol., 1956, 55, 127-142.

ZAJONC, R. B. The effects of feedback and probability of group success on individual and group performance. Hum. Relat., 1962, 15, 149-163.

ZAJONC, R. B., ANd Smoke, W. H. Redundancy in task assignments and group performance. Psychometrika, 1959, 24, 361-370.

ZaJONC, R. B., AND TAYLOR, J. J. The effect of two methods of varying group task difficulty on individual and group performance. Hum. Relat., 1963, 16, 359-368. 